\title{
Estudio exploratorio sobre nociones de ciencias de profesores/as en formación de una carrera de pedagogía en ciencias con mención en física, química y biología
}

\section{Exploratory study on science notions of trainee teachers in science pedagogy with a major in physics, chemistry and biology}

\author{
${ }^{1}$ Darlitt Meza, ${ }^{2}$ Catalina Garrido, ${ }^{3}$ Aaron Álvarez \& ${ }^{4}$ Yefrin Ariza
}

Meza, D., Garrido, C., Álvarez, A., \& Ariza, Y. (2021). Estudio exploratorio sobre nociones de ciencias de profesores/as en formación de una carrera de pedagogía en ciencias con mención en física, química y biología. Revista Convergencia Educativa, (10-extra), diciembre, 74-87. https://doi.org/10.29035/rce.s10.74

[Recibido: 30 enero, 2021 / Aceptado: 06 octubre, 2021]

\begin{abstract}
RESUMEN
En este trabajo exploramos algunas de las ideas que tiene un grupo de 38 estudiantes de una carrera de pedagogía en ciencias con mención en física, química y biología, sobre la dinámica, actividad y producción científica. Este trabajo exploratorio busca promover reflexiones sobre la importancia de intervenir en dichas ideas a fin de contribuir en la construcción de una imagen de ciencia más reciente y enriquecida en los futuros y las futuras docentes de ciencias.
\end{abstract}

Palabras clave: imágenes de ciencia, naturaleza de la ciencia, conocimiento científico.

Temática: Didáctica de las Ciencias, Naturaleza de la ciencia.

* Este artículo fue presentado en las jornadas Internacional de Investigación en Didáctica y Formación Inicial Docente JIIDFID_UCM 2020.

\footnotetext{
1 Estudiante de Pedagogía en Ciencias, Facultad de Ciencias Básicas, Universidad Católica del Maule. Correo electrónico: mezaopazo@gmail.com

${ }^{2}$ Estudiante de Pedagogía en Ciencias, Facultad de Ciencias Básicas, Universidad Católica del Maule. Correo electrónico: catalina.garrido2001@gmail.com

${ }^{3}$ Estudiante de Pedagogía en Ciencias, Facultad de Ciencias Básicas, Universidad Católica del Maule. Correo electrónico: alvarez.silva.aaron@gmail.com

${ }^{4}$ Académico, Facultad de Ciencias Básicas, Universidad Católica del Maule. Correo electrónico: https://orcid.org/0000-0002-1993-7245 | aariza@ucm.cl
} 
Meza, D., Garrido, C., Álvarez, A., \& Ariza, Y. (2021). Estudio exploratorio sobre nociones de ciencias de profesores/as en formación de una carrera de pedagogía en ciencias con mención en física, química y biología. Revista Convergencia Educativa, (10-extra), diciembre, $74-87$. https://doi.org/10.29035/rce.s10.74

\begin{abstract}
In this paper we explore some of the ideas that a group of 38 students of a science pedagogy degree with mention in physics, chemistry and biology have about the dynamics, activity and scientific production. This exploratory work seeks to promote reflections on the importance of intervening in these ideas in order to contribute to the construction of a more recent and enriched image of science in future science teachers.
\end{abstract}

Key words: images of science, nature of science, scientific knowledge.

Themes: Didactics of science, Nature of Science.

\title{
1. INTRODUCCIÓN
}

Entre la investigación actual de la didáctica de las ciencias experimentales, la línea de investigación conocida como "Naturaleza de la Ciencia" (o NOS por sus siglas en inglés) se ha constituido como una de las líneas de mayor dinámica y producción en nuestra disciplina en los últimos años (Adúriz, 2020). Dicha línea se ocupa de identificar, caracterizar e intervenir en las nociones de ciencias de estudiantes, profesores y profesoras. Bajo la idea principal que las nociones que tienen las y los profesores de ciencias influyen sobre las actitudes, acciones y conocimientos que tienen las y los estudiantes acerca del conocimiento científico, ya sea como actividad o producto. Son, por tanto, las ideas que tienen los y las futuras docentes de ciencias acerca del conocimiento científico, un objeto de investigación relevante para la didáctica contemporánea (Leung, 2020).

En este trabajo presentamos los resultados de la aplicación de un cuestionario, enfocado a un grupo de estudiantes de una carrera de pedagogía en ciencias con mención en física, química y biología de la región del Maule (Chile), que buscó identificar el grado de acuerdo con ciertas ideas sobre la ciencia. Para ello, identificamos ciertas afirmaciones sobre la ciencia que, tanto desde la filosofía de la ciencia como desde la didáctica de las ciencias, suelen vincularse con visiones de la ciencia que podrían denominarse “deformadas” (López \& Furió, 2017).

Los resultados principales de este estudio exploratorio muestran un alto grado de acuerdo con dichas afirmaciones (es decir, con visiones deformadas del conocimiento científico) y, en ese sentido, podrían considerarse como "evidencia empírica" acerca de su existencia y de la necesidad de elaborar estrategias de intervención o formación que aborden dichas ideas y posibiliten la construcción de nociones de ciencias más actuales.

\subsection{La enseñanza de las ciencias y el conocimiento metateórico implícito}

En la actualidad, la didáctica de las ciencias experimentales suele reconocer que "enseñar ciencias" no se trata exclusivamente de fomentar la memorización de leyes científicas o las formulaciones matemáticas con las cuales se les suele identificar. Dicha forma de enseñanza que podríamos caracterizar como "tradicional", “[...] suele transmitir una imagen de la ciencia académica del pasado -la que ha producido los conceptos que se incluyen en el currículo-" (Acevedo et al., 2005, p. 131). Hoy en día, y desde hace aproximadamente 30 
Meza, D., Garrido, C., Álvarez, A., \& Ariza, Y. (2021). Estudio exploratorio sobre nociones de ciencias de profesores/as en formación de una carrera de pedagogía en ciencias con mención en física, química y biología. Revista Convergencia Educativa, (10-extra), diciembre, $74-87$. https://doi.org/10.29035/rce.s10.74

años, se reconoce que en la enseñanza de las ciencias se filtra la enseñanza de otros aspectos igualmente relevantes, pero ya no asociados al contenido científico mismo, sino a la forma en la cual se entiende la actividad científica, por ejemplo, la construcción del conocimiento científico, cambios en el tiempo, formas de representación o las maneras en las que se relacionan con la sociedad o la cultura (Pavón \& Cuellar, 2017).

Las reflexiones que se realizan alrededor de los aspectos principales de la actividad científica (productos, métodos, cambios en el tiempo y relaciones con la cultura y sociedad) constituyen lo que se entiende como 'conocimiento metacientífico' (historia de la ciencia, filosofía de la ciencia ${ }^{1}$, sociología de la ciencia). En consonancia con otros autores (Matthews, 1994, Adúriz, 2005, Pavón \& Cuellar, 2017, López \& Furió, 2017) consideramos que la enseñanza de dichas reflexiones contribuye para alcanzar el objetivo actual de una educación científica de calidad para todos y todas. En este sentido, el conocimiento metacientífico permite consolidar, la que se conoce como una 'imagen de ciencia y de científico' valiosa (Adúriz \& Ariza, 2013).

Por lo que, para establecer este tipo de imagen de ciencia más actual y enriquecida, es indispensable que en las acciones docentes tanto de profesores y profesoras de ciencias en ejercicio, profesores en formación inicial y formadores de formadores, se incluyan reflexiones de segundo orden incorporando una mirada fuertemente metateórica (historia, filosofía y enseñanza de la ciencia) que acompañe a la reflexión de carácter didáctico (Adúriz \& Ariza, 2012) vinculada principalmente a concepciones de ciencia actuales (Ariza et al., 2016).

Adúriz (2001) reconoce tres finalidades de una educación científica fuertemente basada en disciplinas metateóricas: la intrínseca, en donde (principalmente) la filosofía e historia de la ciencia facilitan una visión más amplia y desde distintas perspectivas sobre la ciencia que se enseña y aprende; la cultural, en donde la filosofía y la historia de la ciencia permiten acercar la ciencia a la ciudadanía y así contribuir a erigir una visión más humanista; y la instrumental, en la cual la filosofía e historia de la ciencia funcionan como "herramientas auxiliares" para facilitar la comprensión de contenidos científicos. Cada una de estas finalidades se adhiere al propósito de una "alfabetización científica" para que la ciudadanía sea capaz de:

[...] dar sentido a los fenómenos naturales por medio de modelos teóricos, tomar decisiones informadas en asuntos socio-científicos que los atañen, incorporar competencias tales como el pensamiento crítico, la autonomía intelectual, la solidaridad y el cuidado del medio ambiente, valorar las ciencias como producto cultural humano, y establecer una vigilancia ética sobre la relevancia y limitaciones de la ciencia (Acevedo, 2004).

Es, por tanto, necesario el uso de perspectivas desde la NOS que sean incorporadas de manera significativa y explicita (en conjunto con el contenido de las ciencias) en los diversos niveles de la educación

1 Usamos aquí "filosofía de la ciencia" como sinónimo de "epistemología”, en el sentido de una reflexión de segundo orden sobre la ciencia. 
Meza, D., Garrido, C., Álvarez, A., \& Ariza, Y. (2021). Estudio exploratorio sobre nociones de ciencias de profesores/as en formación de una carrera de pedagogía en ciencias con mención en física, química y biología. Revista Convergencia Educativa, (10-extra), diciembre, 74-87. https://doi.org/10.29035/rce.s10.74

científica (Adúriz \& Ariza, 2012), lo cual, presupone, una formación metacientífica adecuada y actual por parte del profesorado de ciencias (Adúriz, 2020): en ejercicio, en formación y formadores de formadores.

\section{2. ¿Qué epistemología es necesaria para la formación de profesores/as de ciencias?}

Si bien no es posible aún dar una respuesta definitiva a esta pregunta, las investigaciones actuales en la didáctica de las ciencias experimentales han emparentado a la enseñanza tradicional (centrada en la memorización de fórmulas y enunciados) con las epistemologías clásicas de la ciencia, de corte lógicopositivista. Dichas posturas provenientes de los años veinte a cincuenta del siglo pasado, se suelen caracterizar por reducir a la ciencia a las enunciaciones lingüístico-axiomáticas de las leyes, y enfocarse en el análisis (lógico o formal) de dichos constructos científicos, por encima de los estudios relacionados con la construcción y creación de teorías o sus determinantes históricos.

Las llamadas ideas "ingenuas" o "deformadas" sobre el conocimiento científico (López \& Furió, 2017) suelen relacionarse (aunque algunas veces de manera errónea) con las ideas sostenidas del positivismo lógico, y son construidas por los y las estudiantes desde edades tempranas, afianzándose por la enseñanza tradicional mientras se avanza en los niveles de escolarización.

Dentro de estas ideas se encuentra una gran gama de estereotipos hacia las personas que se dedican al desarrollo de la actividad científica, caracterizándolas según su inteligencia y aspecto, tales como: la actividad científica es desarrollada especialmente por hombres (blancos en su mayoría); que los científicos trabajan sólo en disciplinas como Física, Química, Biología o Medicina; que poseen una inteligencia superior (aunque también aparece la idea del "científico loco") o que los científicos suelen alejarse de la sociedad encerrados en sus laboratorios (usualmente de química). Asimismo, se encuentran ideas referentes al poder de la ciencia, y su base se halla en el llamado "método científico", el cual se sugiere como rígido e infalible basado en la observación neutra y que asegura la consecución de teorías verdaderas (Pujalte et al., 2014). En general, es posible que estas ideas no solo sean influenciadas mediante experiencias e intercambios sociales y culturales del estudiante, sino también, de forma más significativa, por los medios de comunicación (televisión, cine, cómics, publicidad, etc.) y la propia educación.

Dichas creencias afectan a la enseñanza y se convierten en verdaderos obstáculos cuando se pretende generar y formar a los y las estudiantes desde una ciencia más humana, cuyo campo de trabajo sea accesible para todos y todas, decidan o no seguir una carrera científica (Porlán, 1989). La ciencia no es una actividad reservada para una minoría con características especiales, sino, más bien es posible caracterizarla como una actividad intrínsecamente humana (Echeverría, 1998), hecha por y para humanos, y así, de manera intrínseca, puede ser inexacta, aproximativa y cambiante (Adúriz \& Ariza, 2013)

En general, las principales características de una imagen de ciencia estereotipada (Pujalte et al., 2014) recaen en ideas como, por ejemplo:

- Un monismo metodológico: relacionado con la prevalencia de un único método científico y cuyo seguimiento "aseguraría" la construcción de teorías fiables. Algo que la propia historia de la ciencia 
Meza, D., Garrido, C., Álvarez, A., \& Ariza, Y. (2021). Estudio exploratorio sobre nociones de ciencias de profesores/as en formación de una carrera de pedagogía en ciencias con mención en física, química y biología. Revista Convergencia Educativa, (10-extra), diciembre, $74-87$. https://doi.org/10.29035/rce.s10.74

permitiría criticar, y en la que se pueden reconocer metodologías variadas donde incluso la imaginación y la suerte han jugado un papel central.

- La neutralidad u objetividad de la observación: sostiene que las y los científicas/os pueden liberarse de todas sus ideas y marcos conceptuales previos al realizar las observaciones. Dicha neutralidad fue ya refutada tanto desde los análisis gestálticos como desde los análisis provenientes de la propia filosofía de la ciencia, los cuales introducen la noción de "carga teórica de la observación".

- La idea de "teoría" como subordinada a la noción de "ley científica" (sostener que una teoría, si se comprueba, se convierte en ley), la cuál ha sido rebatida incluso desde los análisis actuales de la filosofía de la ciencia, los cuales sitúan a las teorías como los productos principales de la actividad científica, las cuales tienen como uno de sus componentes, ni el principal, ni el definitivo, ni el identitario, a sus leyes; si, en cambio, sus modelos (Ariza et al., 2016).

- Correspondencia entre teoría y realidad (o realismo ingenuo), la cual está en inconsistencia con los análisis filosóficos actuales acerca del carácter representacional y aproximativo de las construcciones científicas, alejándose de ideas relacionadas con las teorías como copias exactas de la realidad (Ariza et al., 2016).

Si bien las posturas acerca de qué epistemología incluir en la formación de profesores de ciencias se mantiene en discusión, el consenso es más o menos generalizado en cuanto a que la epistemología clásica se relaciona fuertemente con la enseñanza tradicional de corte memorístico (Lederman, 1992), mientras que las posturas más recientes (incluso posteriores a Kuhn) relacionadas con la concepción semántica de las teorías (Adúriz \& Ariza, 2013) brindan oportunidades interesantes para pensar la ciencia en las aulas de clases. Es decir, es posible reconocer una tendencia mayoritaria a acudir a posturas modelo-teóricas de la ciencia (Ariza et al., 2016, 2020, Izquierdo, 2004).

\section{METODOLOGÍA}

Se siguió una metodología cualitativa del tipo exploratoria que consistió en la aplicación de una encuesta tipo "Likert" con ocho preguntas referidas a diversos aspectos del conocimiento científico. Cabe destacar que dicho instrumento para identificar creencias relacionadas con nociones de la actividad científica pertenecientes a concepciones ingenuas o estereotipadas. En ese sentido, la encuesta buscó identificar el grado de acuerdo o desacuerdo que tienen las y los estudiantes sobre ideas de la ciencia que podrían entenderse como "superadas" por los estudios metacientíficos actuales:

1. Los experimentos son los que deciden si una teoría es rechazada o aprobada (papel del experimento).

2. Lo que caracteriza a la práctica científica es el seguimiento estricto del método científico (criterio de demarcación).

3. Los grandes avances en la ciencia se han dado gracias al trabajo e investigación de los hombres. Ellos tienen mejores habilidades científicas que las mujeres (ciencia y género). 
Meza, D., Garrido, C., Álvarez, A., \& Ariza, Y. (2021). Estudio exploratorio sobre nociones de ciencias de profesores/as en formación de una carrera de pedagogía en ciencias con mención en física, química y biología. Revista Convergencia Educativa, (10-extra), diciembre, 74-87. https://doi.org/10.29035/rce.s10.74

4. Cuanto más comprobamos una teoría, más nos acercamos a la realidad (representación y correspondencia).

5. Los científicos siempre inician su actividad observando varios casos particulares de un fenómeno para luego inducir leyes universales (metodología de la ciencia).

6. Las observaciones que hacen los/as científicos/as son objetivas y neutras. (papel de la observación)

7. Una ley, es una teoría que se comprobó (estructura científica).

8. La ciencia avanza por acumulación de teorías verdaderas (cambio científico).

La muestra fue no probabilística, ya que se usó como criterio de exclusión que todos los y las estudiantes participantes estuvieran cursando actualmente una carrera de Pedagogía en Ciencias con Mención en la región del Maule, de nueve semestres de duración y con plan común los tres primeros. Posteriormente, cada estudiante elige una mención en la cual se especializa, completando los semestres restantes. Dichas menciones se dividen en tres: Física, Química y Biología.

La participación de las y los estudiantes fue voluntaria y consentida; accedieron a participar del estudio luego de aceptar un consentimiento informado para resguardar datos que podrían individualizarse, con una noción clara respecto a los fines por los cuales se estaba llevando a cabo dicho instrumento.

Este instrumento evaluativo recopiló los datos de 37 estudiantes de pregrado con un rango de edad entre los 17 y 27 años. Siendo 23 (63.89\%) mujeres y 13 estudiantes (36,11\%) hombres.

\section{RESULTADOS}

A continuación, se presentan los resultados para cada una de las preguntas aplicadas:

1. Los experimentos son los que deciden si una teoría es rechazada o aprobada.

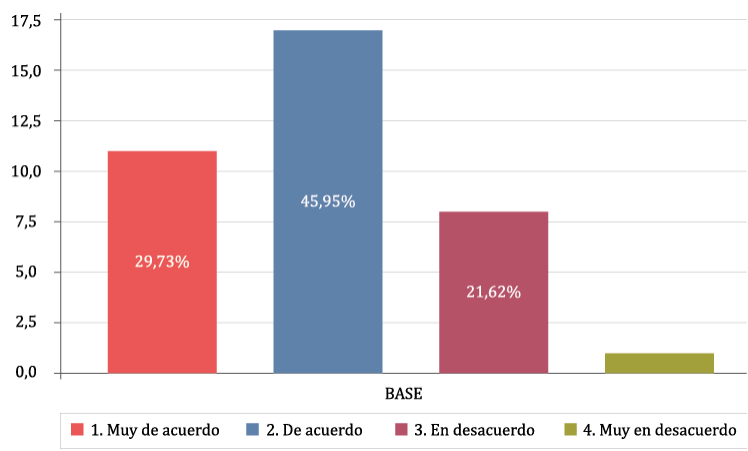

Gráfico 1. Respuestas pregunta 1. 
Meza, D., Garrido, C., Álvarez, A., \& Ariza, Y. (2021). Estudio exploratorio sobre nociones de ciencias de profesores/as en formación de una carrera de pedagogía en ciencias con mención en física, química y biología. Revista Convergencia Educativa, (10-extra), diciembre, 74-87. https://doi.org/10.29035/rce.s10.74

2. Lo que caracteriza a la práctica científica es el seguimiento estricto del método científico.

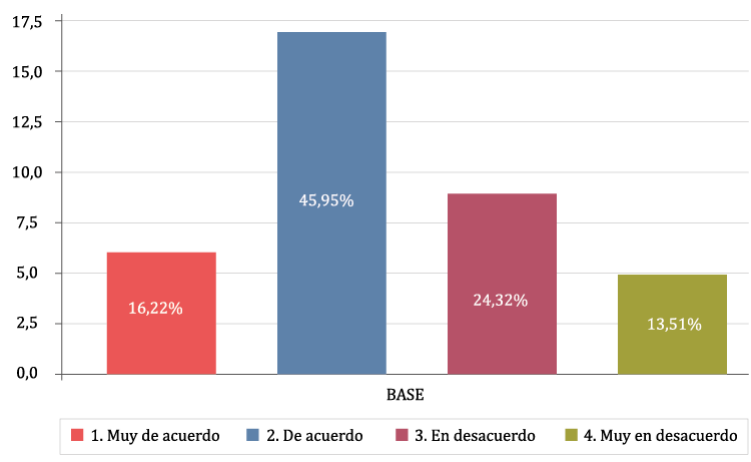

Gráfico 2. Respuestas pregunta 2.

3. Los grandes avances en la ciencia se han dado gracias al trabajo e investigación de los hombres. Ellos tienen mejores habilidades científicas que las mujeres.

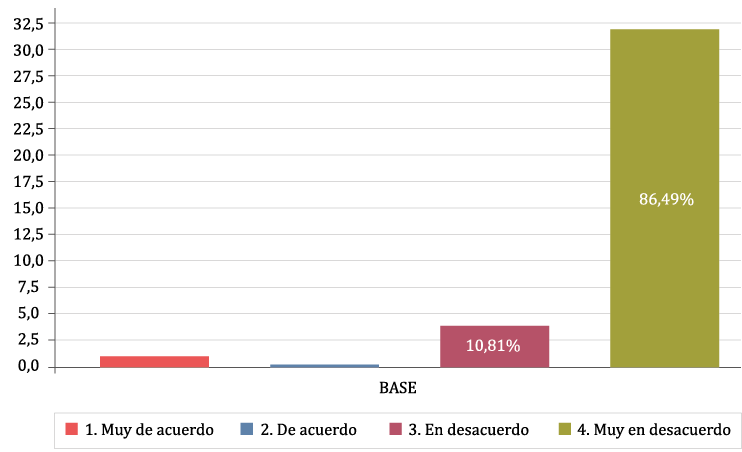

Gráfico 3. Respuestas pregunta 3.

4. Cuanto más comprobamos una teoría, más nos acercamos a la realidad.

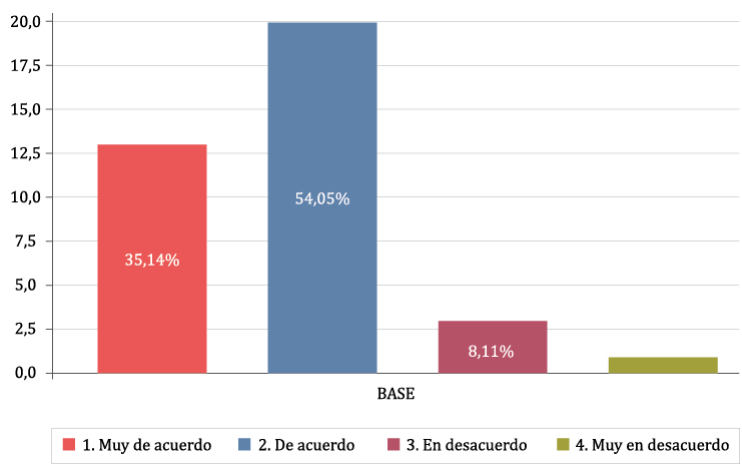

Gráfico 4. Respuestas pregunta 4. 
Meza, D., Garrido, C., Álvarez, A., \& Ariza, Y. (2021). Estudio exploratorio sobre nociones de ciencias de profesores/as en formación de una carrera de pedagogía en ciencias con mención en física, química y biología. Revista Convergencia Educativa, (10-extra), diciembre, 74-87. https://doi.org/10.29035/rce.s10.74

5. Los científicos siempre inician su actividad observando varios casos particulares de un fenómeno para luego inducir leyes universales.

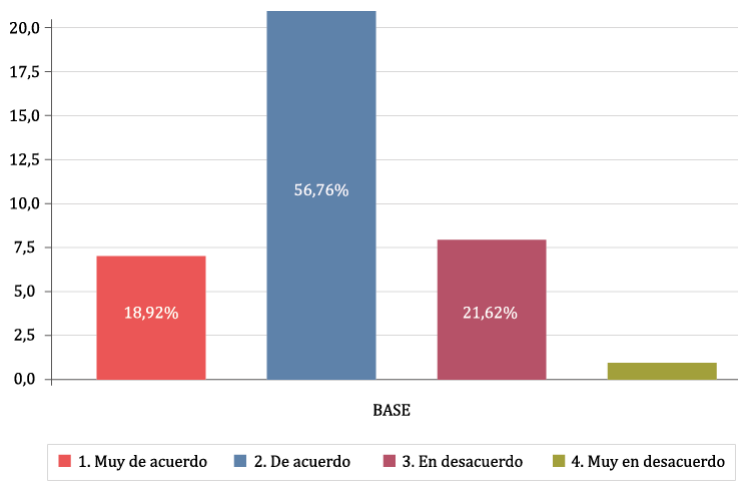

Gráfico 5. Respuestas pregunta 5.

6. Las observaciones que hacen los/as científicos/as son objetivas y neutras.

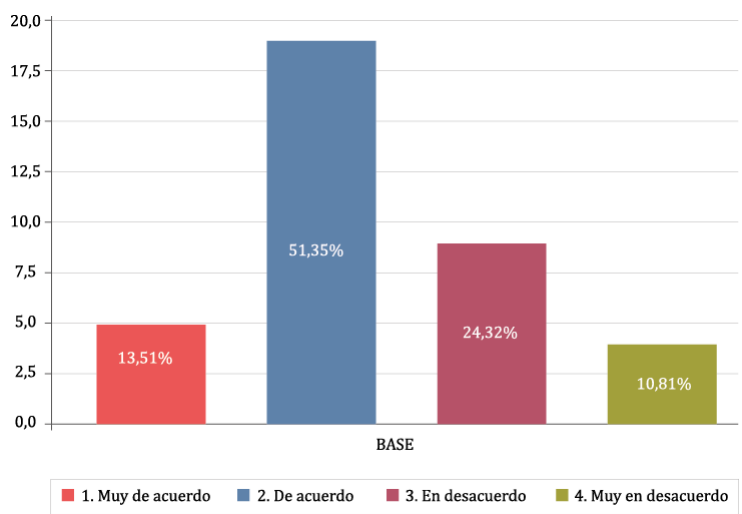

Gráfico 6. Respuestas pregunta 6.

7. Una ley, es una teoría que se comprobó.

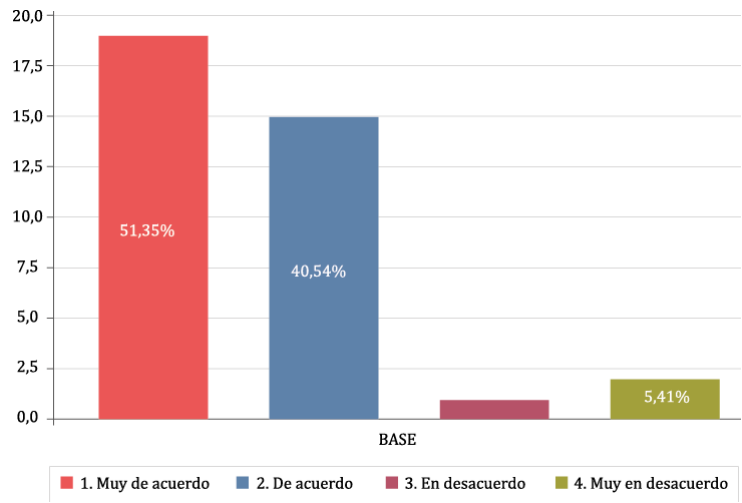

Gráfico 7. Respuestas pregunta 7. 
Meza, D., Garrido, C., Álvarez, A., \& Ariza, Y. (2021). Estudio exploratorio sobre nociones de ciencias de profesores/as en formación de una carrera de pedagogía en ciencias con mención en física, química y biología. Revista Convergencia Educativa, (10-extra), diciembre, 74-87. https://doi.org/10.29035/rce.s10.74

8. La ciencia avanza por acumulación de teorías verdaderas.

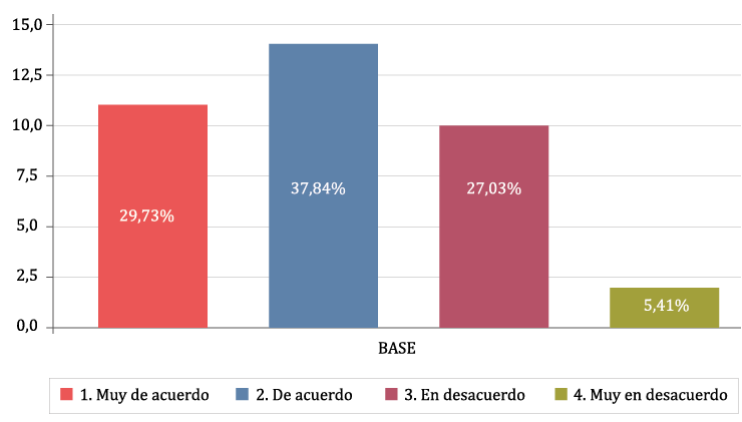

Gráfico 8. Respuestas pregunta 8.

\section{ANÁLISIS}

Los resultados generales muestran una tendencia clara hacia la aceptación de las afirmaciones presentadas, representada una elección mayoritaria de las opciones "de acuerdo" o "muy de acuerdo", con excepción la afirmación relacionada con la ciencia y género, en la cual existió una amplia oposición (97,3\% en desacuerdo o muy en desacuerdo). En consonancia con ello, las y los estudiantes manifiestan imágenes de ciencia emparentadas con posiciones estereotipadas e ingenuas usualmente emparentadas (cómo se mencionó en líneas anteriores) a las escuelas de la filosofía de la ciencia de la primera mitad del siglo XX, realzando criterios referentes al papel del experimento (75.74\%), representación y correspondencia (89.19\%), metodología de las ciencias (75.68\%) y estructura científica (91.89\%).

En ese sentido, y siguiendo la idea de que aquellas concepciones sobre el conocimiento científico que mantiene el profesorado de ciencias naturales repercuten incisivamente sobre ideas, representaciones y actitudes de los y las estudiantes; sería posible afirmar que se continúan reforzado imágenes de ciencia ya superadas por las investigaciones actuales de la filosofía de la ciencia y que, consecuentemente, podrían fomentar la construcción de imágenes de ciencia inadecuadas en las futuras aulas de clase del profesorado en formación.

\section{CONCLUSIONES}

Si bien la muestra es acotada, y por ende los resultados podrían considerarse poco representativa, están en consonancia con evidencia identificada en investigaciones internacionales de la didáctica de las ciencias (Pujalte et al., 2014). En este sentido, y a nivel nacional, un estudio similar y con resultados coincidentes fue, por ejemplo, el realizado por el equipo de investigación de Mario Quintanilla (Quintanilla et al., 2006) que buscó caracterizar las concepciones metateóricas sobre la naturaleza de la ciencia y su enseñanza, que poseen los profesores.

Al mismo tiempo, consideramos que este estudio es una aproximación a la caracterización acerca de las concepciones de ciencia que tienen en general los profesores de ciencias en formación, y, en consecuencia, podría entenderse como un insumo para futuros estudios que busquen intervenir activamente en las imágenes de ciencia y de científicos de los profesores en formación. 
Meza, D., Garrido, C., Álvarez, A., \& Ariza, Y. (2021). Estudio exploratorio sobre nociones de ciencias de profesores/as en formación de una carrera de pedagogía en ciencias con mención en física, química y biología. Revista Convergencia Educativa, (10-extra), diciembre, 74-87. https://doi.org/10.29035/rce.s10.74

La importancia de este tipo de estudios recae en los efectos que una imagen inadecuada de las ciencias puede tener en la educación, generando verdaderos obstáculos para la enseñanza de una ciencia más humana e incluso para el desarrollo de las distintas carreras científicas.

La continua necesidad de actualización del profesorado, en conjunto con el diagnóstico y tratamiento de las imágenes de ciencia, a fin de promover el pensamiento crítico en torno a nuestros futuros y futuras profesores y profesoras de ciencias, se pone en evidencia cuando encontramos resultados como los expuestos aquí. Si bien, fue un acercamiento exploratorio muy básico, es posible identificar el requerimiento de generar instancias de formación metateoría del profesorado, coincidente, a su vez, con la presencia explícita de dichos contenidos de carácter "meta" en las bases curriculares de nuestro país. En la misma línea, es relevante pensar y actuar ante la constante transmutación de la sociedad, en donde debemos posicionar a la ciencia como una herramienta que tenga un rol trascendental en la toma de decisiones, científicamente y metacientíficamente informadas, que influyan en la cotidianidad de la ciudadanía. 
Meza, D., Garrido, C., Álvarez, A., \& Ariza, Y. (2021). Estudio exploratorio sobre nociones de ciencias de profesores/as en formación de una carrera de pedagogía en ciencias con mención en física, química y biología. Revista Convergencia Educativa, (10-extra), diciembre, 74-87. https://doi.org/10.29035/rce.s10.74

\section{ANEXO}

\section{Pauta de revisión de artículos}

\begin{tabular}{|c|c|c|c|c|}
\hline INDICADORES & Deficiente & Aceptable & Bueno & Muy bueno \\
\hline \multicolumn{5}{|l|}{ ASPECTOS DE CONTENIDO } \\
\hline $\begin{array}{l}\text { Relevancia (grado de aporte a la comunidad } \\
\text { científica). }\end{array}$ & & $\mathrm{x}$ & & \\
\hline Método (acorde y coherente al tipo de artículo). & $\mathrm{x}$ & & & \\
\hline $\begin{array}{l}\text { Consistencia interna (fundamentación, } \\
\text { objetivos, método, resultados o conclusiones, en } \\
\text { función al tipo de artículo). }\end{array}$ & $\mathrm{x}$ & & & \\
\hline Resultados o Conclusiones. & $\mathrm{x}$ & & & \\
\hline \multicolumn{5}{|l|}{ ASPECTOS FORMALES } \\
\hline $\begin{array}{l}\text { Claridad lógico gramatical del texto (redacción } \\
\text { coherente y ajustada al tipo de artículo). }\end{array}$ & & $\mathrm{x}$ & & \\
\hline $\begin{array}{l}\text { Presentación (actualizada acorde a los criterios } \\
\text { vigentes en la comunidad científica y a lo } \\
\text { estipulado por Comité Editorial). }\end{array}$ & & $\mathrm{x}$ & & \\
\hline $\begin{array}{l}\text { Citas de referencias en el texto (cumple con los } \\
\text { criterios vigentes, para cada caso en particular). }\end{array}$ & & $\mathrm{x}$ & & \\
\hline $\begin{array}{l}\text { Referencias al final de texto (responden a } \\
\text { normativa APA y son coincidentes con las citas } \\
\text { en el texto). }\end{array}$ & & & $\mathrm{x}$ & \\
\hline
\end{tabular}

Si lo desea, puede incorporar en este espacio otras observaciones o sugerencias sobre el artículo revisado:

\section{OBSERVACIONES}

Si bien el artículo trata un tema novedoso y adecuado a la Didáctica de las Ciencias, posee falencias importantes: Aspectos formales:

Posee errores gramaticales y de uso de signos de puntuación.

El lenguaje usado tiende a sintetizar la idea de tal manera que se pierde el sentido de lo que se desea explicar.

Si bien las citas bibliográficas están relacionadas con lo que se aborda en el escrito, son demasiado antiguas. Más del $90 \%$ de las citas son de una antigüedad mayor a 5 años. Hay ideas que el autor indica como "reciente" que es sustentada en un estudio del año 2016, por ejemplo, lo cual carece de sentido en el 2021.

En ocasiones los autores indican ideas que no son sustentadas por referencias bibliográficas.

Aspectos de contenido:

Si bien es un estudio básico (así lo definen los autores) es un buen trabajo exploratorio. Los resultados y conclusiones responden al objetivo que se plantea al inicio del artículo, no obstante, le falta robustez al análisis de los resultados y a las conclusiones. Creo necesario incluir el contraste de estos resultados con los obtenidos por otros autores en estudios similares.

El estudio se sustenta en un instrumento tipo encuesta, que no está validada por expertos. Tampoco se indica si se trata de un instrumento publicado en otro trabajo o adaptado. 
Meza, D., Garrido, C., Álvarez, A., \& Ariza, Y. (2021). Estudio exploratorio sobre nociones de ciencias de profesores/as en formación de una carrera de pedagogía en ciencias con mención en física, química y biología. Revista Convergencia Educativa, (10-extra), diciembre, $74-87$. https://doi.org/10.29035/rce.s10.74

\section{REFERENCIAS BIBLIOGRÁFICAS}

Acevedo J. A., Vázquez, A., Martín, M., Oliva, J., Acevedo, P., Paixão, M., \& Manassero, M. A. (2005). Naturaleza de la ciencia y educación científica para la participación ciudadana: Una revisión crítica, Revista Eureka sobre Enseñanza y Divulgación de las Ciencias, 2(2), 121-140. https://revistas.uca.es/index.php/eureka/article/view/3912

Acevedo-Díaz, J.A. (2004) Reflexiones sobre las Finalidades de la Enseñanza de las Ciencias: Educación Científica para la Ciudadanía, Revista Eureka sobre Enseñanza y Divulgación de las Ciencias, 1(1), 316. https://revistas.uca.es/index.php/eureka/article/view/3968

Adúriz-Bravo, A. (2020). Contributions to the nature of science. Scientific investigation as inquiry, modeling, and argumentation. En C. El-Hani, M. Pietrocola, E. Mortimer, \& M. Otero (Eds.). Science Education Research in Latin America (Cultural and Historical Perspectives on Science Education) (pp. 394425). Netherlands: Brill | Sense.

Adúriz-Bravo, A. (2005). Una introducción a la naturaleza de la ciencia. La epistemología en la enseñanza de las ciencias naturales, Fondo de Cultura Económica.

Adúriz-Bravo, A. (2001). Integración de la epistemología en la formación del profesorado de ciencias. [Tesis Doctoral, Universitat Autònoma de Barcelona]. https://www.tdx.cat/bitstream/handle/10803/4695/aab1de3.pdf

Adúriz-Bravo, A., \& Ariza, Y. (2013). Las imágenes de ciencia y de científico. Una puerta de entrada a la naturaleza de la ciencia. En A. Adúriz-Bravo, M. Dibarboure, \& S. Ithurralde (coords.): El quehacer del científico al aula. Pistas para pensar (pp. 13-20). FUM-TEP/Fondo Editorial QUEDUCA.

Adúriz-Bravo, A., \& Ariza, Y. (2012). Importancia de la filosofía y de la historia de la ciencia en la enseñanza y en el aprendizaje de las ciencias. En Z. Monroy-Nasr, R. León-Sánchez, \& G. Alvarez Díaz de León, (Comp). Enseñanza de la Ciencia (pp. 79-92). DGAPA \& Facultad de Psicología, Universidad Autónoma de México.

Ariza, Y., \& Adúriz-Bravo, A. (2011). La nueva filosofía de la ciencia y la concepción semántica de las teorías científicas en la didáctica de las ciencias naturales. Educación en Ciencias Matemáticas y Experimentales, 2, 55-66.

Ariza, Y., Lorenzano, P., \& Adúriz-Bravo, A. (2016). Meta-theoretical contributions to the constitution of a model-based didactics of science. Science \& Education, 25(7-8), 747-773. https://bibliotecadigital.exactas.uba.ar/collection/paper/document/paper_09267220_v25_n78_p747_Ariza

Ariza, Y., Lorenzano, P., \& Adúriz-Bravo, A. (2020). Bases modeloteóricas para la ciencia escolar: la noción de "comparabilidad empírica". Estudios Pedagógicos, 46(2), 447-469. https://doi.org/10.4067/S0718-07052020000200447 
Meza, D., Garrido, C., Álvarez, A., \& Ariza, Y. (2021). Estudio exploratorio sobre nociones de ciencias de profesores/as en formación de una carrera de pedagogía en ciencias con mención en física, química y biología. Revista Convergencia Educativa, (10-extra), diciembre, 74-87. https://doi.org/10.29035/rce.s10.74

Echeverría, J. (1998). Ciencia y valores: propuestas para una axionomía de la ciencia. Contrastes. Revista $\begin{array}{lllll}\text { Internacional de } \quad \text { Filosofía, } & \text { Suplemento }\end{array}$ https://revistas.uma.es/index.php/contrastes/article/view/1540

Izquierdo-Aymerich, M. (2004). Un nuevo enfoque de la enseñanza de la química: contextualizar y modelizar, The Journal of the Argentine Chemical Society, 92(4-6), 115-136. http://www.scielo.org.ar/scielo.php?script=sci_abstract\&pid=S0365-03752004000200013

Lederman, N. (1992). Students' and Teachers' Conceptions of the Nature of Science: A Review of the Research, Journal of Research in Science Teaching, 29(4), 331-359. https://doi.org/10.1002/tea.3660290404

Leung, J.S.C. (2020). A Practice-Based Approach to Learning Nature of Science through Socioscientific Issues. Research and Science Education, 1-27. https://doi.org/10.1007/s11165-020-09942-w

López-Valentín, D., \& Furió, C. (2017). Visiones deformadas de la ciencia en la enseñanza del concepto de elemento químico. Enseñanza de las ciencias, (Extra), 3747-3752. https://core.ac.uk/download/pdf/147042939.pdf

Matthews, M. (1994). Science teaching: The role of history and philosophy of science, Routledge.

Pavón, Z., \& Cuellar, L. (2017). Relaciones entre las concepciones de naturaleza de la ciencia y la tecnología, y de la enseñanza y el aprendizaje de las ciencias de profesores de química en ejercicio. Una primera aproximación al esquema conceptual del profesor. Tecné, Episteme y Didaxis, TED, (41), 17-36. https://doi.org/10.17227/01203916.6030

Porlán, R. (1989). Teoría del conocimiento, teoría de la enseñanza y desarrollo profesional. Las concepciones epistemológicas de los profesores. [Tesis doctoral]. Universidad de Sevilla, Sevilla. https://idus.us.es/handle/11441/85207

Pujalte, A., Bonan, M. L., Porro, S., \& Adúriz-Bravo, A. (2014). Las imágenes inadecuadas de ciencia y de científico como foco de la naturaleza de la ciencia: Estado del arte y cuestiones pendientes. Ciência \& Educação 20(3), 535-548. https://doi.org/10.1590/1516-73132014000300002

Quintanilla, M., Labarrere, A., Santos, M., Cadiz, J., Cuellar, L., Saffer, G., \& Camacho, J. (2006). Elaboración, validación y aplicación preliminar de un cuestionario sobre ideas de la imagen de ciencia y educación científica de profesores en servicio. Boletín de Investigación Educacional, 21(2), 103-132. http://www7.uc.cl/sw_educ/educacion/grecia/plano/html/pdfs/linea_investigacion/Que_Cienci a_Ensenar_IEC/IEC_045.pdf 
Meza, D., Garrido, C., Álvarez, A., \& Ariza, Y. (2021). Estudio exploratorio sobre nociones de ciencias de profesores/as en formación de una carrera de pedagogía en ciencias con mención en física, química y biología. Revista Convergencia Educativa, (10-extra), diciembre, $74-87$. https://doi.org/10.29035/rce.s10.74

\section{Datos de correspondencia}

Yefrin Ariza

Académico, Facultad de Ciencias Básicas

Universidad Católica del Maule. Talca, Chile.

Dirección postal: Avenida San Miguel \#3605. Talca, Chile.

ORCID ID: https://orcid.org/0000-0002-1993-7245

Email: aariza@ucm.cl 